\title{
Extracorporeal membrane oxygenation support in patients with hematologic malignancies: to whom and when?
}

\author{
Junshik Hong ${ }^{1}$ and Chang Hyu Choi ${ }^{2}$
}

Departments of ${ }^{1}$ Internal Medicine and ${ }^{2}$ Cardiothoracic Surgery, Gachon University Gil Medical Center, Incheon, Korea

Received: August 7, 2016

Accepted: January 5, 2017

\section{Correspondence to}

Chang Hyu Choi, M.D.

Department of Cardiothoracic Surgery, Gachon University Gil Medical Center, 21 Namdongdaero 774beon-gil, Namdong-gu, Incheon 21565, Korea

Tel: +82-32-460-3666

Fax: +82-32-46o-2371

E-mail: cch624@gilhospital.com
A3o-year-old male presented with fever, abdominal distension, and a white blood cell (WBC) count of 101,700/mm33. A bone marrow immunophenotyping study showed about $91 \%$ of marrow nucleated cells were lymphoblasts expressing CD45, CD10, $\mathrm{Cd}_{2}, \mathrm{sCD}_{3}, \mathrm{CD}_{4}, \mathrm{CD}_{5}$, and $\mathrm{CD}_{7}$, which was consistent with acute T-lymphoblastic leukemia/lymphoma (T-ALL), medullary type. The patient had a normal karyotype but fluorescence in situ hybridization study revealed CDKN2A 9p21 deletion. At diagnosis, he had no respiratory symptom and chest X-ray (CXR) findings were normal. On day 7 of induction chemotherapy, which was composed of vincristine, prednisolone, daunorubicin, and L-asparaginase, he complained of a cough and right pleuritic chest pain with fever. Despite the use of broad-spectrum antibiotics, the fever persisted, and on day 12, CXR depicted peribronchial consolidation. His symptoms then rapidly aggravated. On day 15, his $\mathrm{PaO}_{2}$ was 53 $\mathrm{mmHg}$ with a $\mathrm{FiO}_{2}$ of 0.8 by a reservoir mask, and his laboratory results were as follows: hemoglobin $8.3 \mathrm{~g} / \mathrm{dL}$, WBCs 840/ $\mathrm{mm}^{3}$ (absolute neutrophil count [ANC] 250/(mm 3 ), and platelets 102,000/mm3; renal and hepatic functions were normal. Due to uncorrectable and progressing hypoxemia, mechanical ventilation after endotracheal intubation was decided. A positive result for Legionella urinary an- tigen raised the possibility of Legionella pneumonia, although no microorganism was reported by culture of any body fluid or by molecular testing for a respiratory infection. Intensive efforts, including escalation of antimicrobial agents and prone positioning, were then taken. However, he failed to maintain $\mathrm{a} \mathrm{PaO}_{2}$ of $\geq 60 \mathrm{mmHg}$ with $\mathrm{FiO}_{2}$ of 1.o. On day 18 , veno-venous extracorporeal membrane oxygenation (ECMO) support was decided; at that time, he had no impairment of cardiac, renal, or hepatic function. Five days later, he escaped from severe neutropenia (ANC $<500 / \mathrm{mm}^{3}$ ), and CXRs showed gradual improvement (Fig. 1). ECMO was removed on day 36 and on day 50 , complete remission of T-ALL was confirmed by bone marrow study and he discharged from hospital. Subsequently, he underwent consolidative chemotherapy and at the time of writing, over 28 months after diagnosis, was surviving.

Kang et al. [1] reported outcomes of ECMO in 15 patients with hematologic malignancies (HMs). This study is valuable because few reports have been issued on ECMO in patients with HM, which are a profoundly immunosuppressed population, but with the possibility of cure. Because all 15 patients died in this previous study, negative impressions are inevitable with regard to ECMO in patients with HM. Howev- 

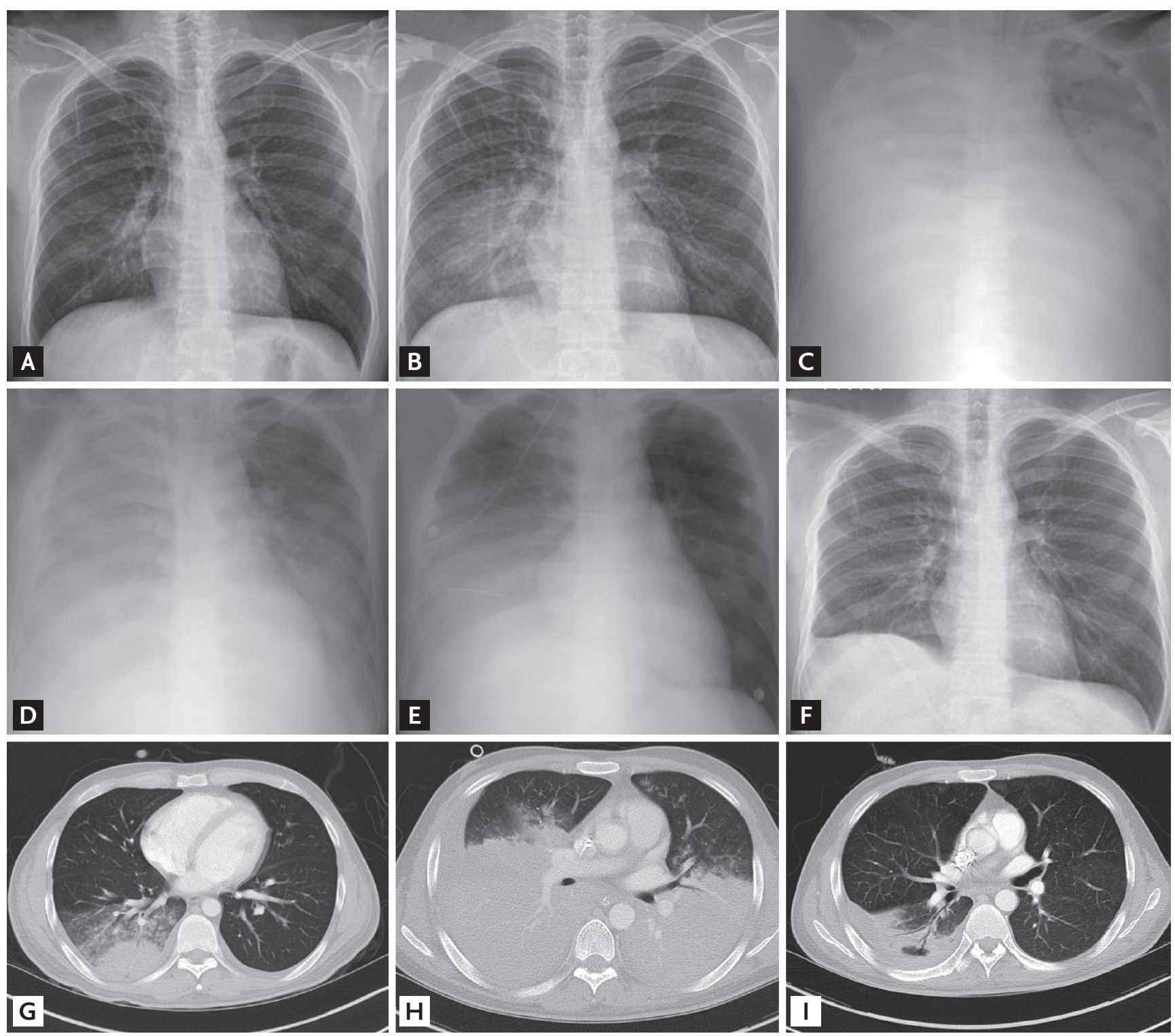

Figure 1. Serial chest X-rays of our patient. (A) At diagnosis. (B) On day 12 of induction chemotherapy. (C) On day 18 , just after the initiation of extracorporeal membrane oxygenation (ECMO). (D) On day 25 and (E) on day 36, immediately after the removal of ECMO. (F) A year after diagnosis. Chest computed tomographic findings. (G) On day 14 of induction chemotherapy. (H) On day 19 and (I) on day 60 of induction chemotherapy.

er, our patient shows potential benefit of ECMO in patients with $\mathrm{HM}$ can be realized if patients are adequately selected [2,3]. Wohlfarth et al. [4] conducted a retrospective analysis of 14 Austrian patients with HM that received ECMO and reported more optimistic outcomes; seven achieved long-term disease-free survival. A comparison of patient characteristics in these two previous studies suggests reasons for the survival difference (०\% vs. 50\%). First, the Korean patients were older (mean $46 \pm$ 13.6 years) than the Austrian patients (median 32; range,
21 to 51). Second, the types of HM included were fundamentally different. In the Korean study, nine patients had acute myeloid leukemia (AML) or myelodysplastic syndrome (MDS), and only one patient had Hodgkin lymphoma. On the other hand, in the Austrian study, eight patients had lymphoma and only one patient had AML. Thus, it appears the inclusion of younger patients and more patients with malignant lymphoma, which are indicators of better survival than AML/MDS, may have contributed to this difference. It might be due to the 
difference of the intensity of chemotherapy (i.e., high dose chemotherapy for AML or ALL, leading to several weeks of myelosuppression vs. chemotherapy for lymphoma such as CHOP [cyclophosphamide, doxorubicin, vincristine, and prednisone] regimen leading to only several days of neutropenia), rather than type of HM per se. One feature shared by these two studies is that patients with ECMO after allogenic stem cell transplantation (allo-SCT) showed extremely poor outcomes; seven Korean and four Austrian patients received ECMO after allo-SCT, and none survived $[1,4]$. The poor outcomes may have been due to a profound immunosuppression. Although the Extracorporeal Life Support Organization (ELSO) guideline suggests major pharmacologic immunosuppression (ANC $<400 \mathrm{~mm}^{3}$ ) is a contraindication [5], we decided to apply ECMO because we expected that the duration of neutropenia would be limited, and therefore, there was some likelihood of improvement. Based on our experience, we suggest the expected duration of immunosuppression should be taken into account when considering ECMO.

Future studies with larger number of patients in various types of HM with different disease severities are needed to define the role of ECMO more precisely.

\section{Conflict of interest}

No potential conflict of interest relevant to this article was reported.

\section{REFERENCES}

1. Kang HS, Rhee CK, Lee HY, et al. Clinical outcomes of extracorporeal membrane oxygenation support in patients with hematologic malignancies. Korean J Intern Med 2015;30:478-488.

2. Aboud A, Marx G, Sayer H, Gummert JF. Successful treatment of an aggressive non-Hodgkin's lymphoma associated with acute respiratory insufficiency using extracorporeal membrane oxygenation. Interact Cardiovasc Thorac Surg 2008;7:173-174.

3. Oto M, Inadomi K, Chosa T, Uneda S, Uekihara S, Yoshida M. Successful use of extracorporeal membrane oxygenation for respiratory failure caused by mediastinal precursor T lymphoblastic lymphoma. Case Rep Med 2014;2014:804917.

4. Wohlfarth P, Ullrich R, Staudinger T, et al. Extracorporeal membrane oxygenation in adult patients with hematologic malignancies and severe acute respiratory failure. Crit Care 2014;18:R20.

5. Delnoij TS, Driessen R, Sharma AS, Bouman EA, Strauch U, Roekaerts PM. Venovenous extracorporeal membrane oxygenation in intractable pulmonary insufficiency: practical issues and future directions. Biomed Res Int 2016;2016:9367464. 